\title{
On solar radius measurements with Danjon astrolabes
}

\begin{abstract}
F. Noël ${ }^{\star}$
Departamento de Astronomía, Universidad de Chile, Casilla 36-D, Santiago, Chile

Received 31 July 2002 / Accepted 5 September 2002

Abstract. During the last 25 years the Danjon astrolabe has been used extensively for measuring the apparent solar diameter. Here we discuss some aspects of the results obtained with the CCD astrolabes of Antalya (Turkey) and Rio de Janeiro (Brazil), and with the visual astrolabes of CERGA (France) and Santiago (Chile). The most extended databases obtained with these astrolabes are those of CERGA and probably Santiago. However, concerning observed variations in time of the solar radius, the results of both stations are discrepant. While the CERGA results show a marginal radius variation in opposing phase with solar magnetic activity, those of Santiago give a significant variation in time, but in phase with solar activity. According to Chollet \& Sinceac (1999) the apparent solar radius variation is identical for all astrolabe measures (except Santiago) and anticorrelated with the magnetic cycle of the Sun. However, we show here that according to published results, the astrolabes of Antalya and Santiago give similar radius variation. With respect to the results of Rio de Janeiro, they show strong internal inconsitencies due probably to instrumental modifications made during its observational campaign. After removing these inconsistencies, the results of Rio de Janeiro become almost identical to those of Santiago. The results of these astrolabes, Antalya, Rio de Janeiro and Santiago, are in agreement with recent results obtained with different observational techniques, which show also that the apparent solar radius would vary in phase with solar magnetic activity.
\end{abstract}

Key words. astrometry - Sun: fundamental parameters - Sun: actvity

\section{Introduction}

Variations in time of the solar radius is a fairly important issue for Astrophysics. If these variations are real, they would constitute a serious challenge to stellar structure theory. According to Spruit (1994), the implications would be much more serious than a mere contradiction between, say, a dynamo theory and observations of the solar cycle. The suspicion that the solar radius could be variable is a motivation for measuring it, and the role of the results is to guide the theory, rather than to verify it (Ribes et al. 1991).

The Danjon astrolabe has been used extensively to measure the apparent solar radius in the last twenty five years. The astrolabe, developed at the end of the forties and the beginning of the fifties at the Paris Observatory by André Danjon (1960), works according to the method of equal altitudes (Débarbat \& Guinot 1970). It was applied very succesfully to the research of the Earth rotation parameters. However, it was shown that it was also a powerful instrument for stellar astrometry (Anguita \& Noël 1969; Fricke 1972). Good results also have been obtained for astrometric observations of planets (Débarbat 1968; Standish et al. 1981; Noël 1987). The excellent astrometric results obtained by the Danjon astrolabe were due partly to the intrinsic qualities of the instrument and partly to the method of equal altitudes (Danjon 1960).

Following the pioneering work of Dr. Francis Laclare at the Centre d'Études et de Recherches en Géodynamique et Astrométrie (CERGA), France, the Danjon astrolabe,

* e-mail: fnoel@das.uchile.cl after some modifications, also was applied to solar astrometry. Astrolabe observations of the Sun give, among other solar parameters, an absolute measurement of the apparent solar radius (Laclare 1983). The work of Laclare was followed by programs of solar observations with modified Danjon astrolabes in São Paulo and Rio de Janeiro, Brazil (Leister \& Benevides Soares 1990; Jilinski et al. 1999), in San Fernando, Spain (Sánchez et al. 1993), in Antalya, Turkey (Golbasi et al. 2001) and in Santiago, Chile (Chollet \& Noël 1993). All these astrolabes, except Santiago, are now equipped with CCD cameras.

\section{Solar radius variations observed at CERGA and Santiago}

The most extended databases of homogeneous solar radius obtained from these programs and comprising continuous results over a lapse equivalent to or greater than a solar cycle, are those of Laclare at CERGA (Laclare et al. 1996) and Noël at Santiago (Noël 2001). However, there is quite a strong disagreement between the results of both series. While the CERGA measurements show a long term variation of the radius of 0.2 amplitude in opposing phase with sunspot number (Laclare et al. 1999; Emilio et al. 2000; Pap et al. 2001), the measurements of Santiago show a significant radius variation of about 0.5 amplitude, but in phase with solar magnetic activity (Noël 2001, 2002; Pap et al. 2001). This disagreement is illustrated in Fig. 1 which shows for the period 1990-1998, the smoothing curves of the variation in time of the observed solar radius and of sunspots numbers. 


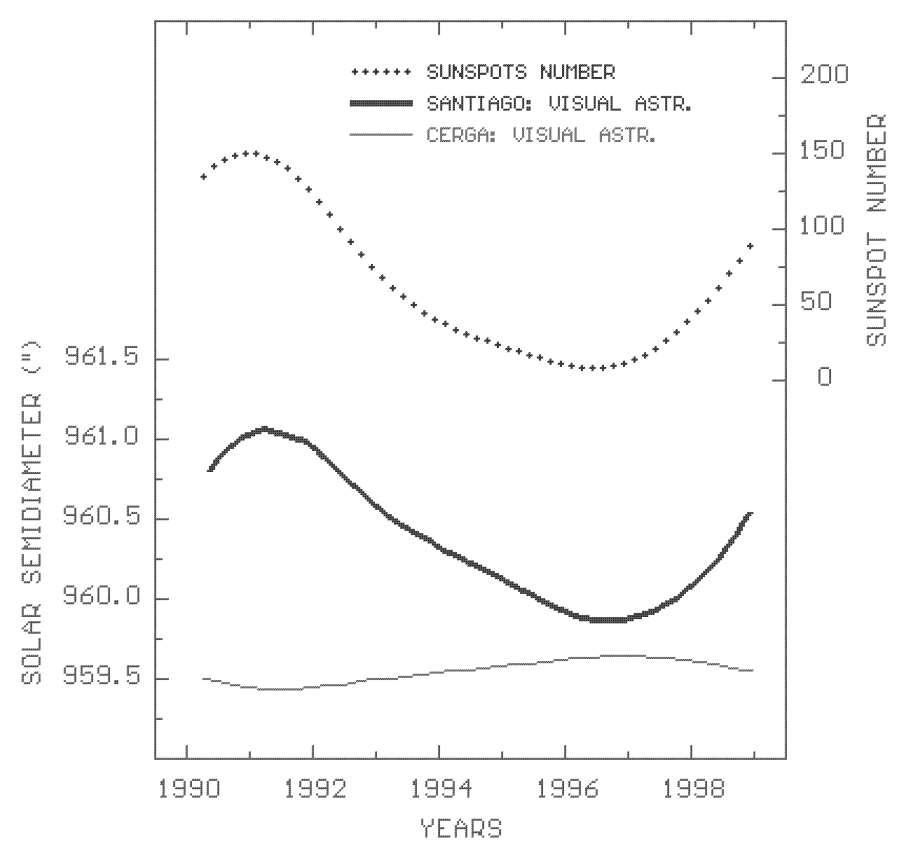

Fig. 1. Variations in time of the solar radius according to the visual astrolabes of CERGA and Santiago, and variation of sunspot number. The curves for Santiago and sunspots are Vondrak fits to monthly means of solar radius, and to monthly sunspot numbers given by SIDC (Noël 2002; Vondrak 1963). The curve for CERGA was adapted from a harmonic fit to individual radius values given in the paper of Pap et al. (2001) (see also Emilio et al. 2000)

Bearing in mind that the solar astrolabes of CERGA and Santiago are essentially similar instruments and both have a similar wide bandpass around $538 \mathrm{~nm}$, it is fair to recognize that this disagreement has been quite disconcerting. However, our view of its probable origin will be discussed in a report in preparation about the definitive results of our program of solar observations made over twelve years by a single observer with the Santiago astrolabe.

Due to its extension, internal consistency and pioneering character, the visual results of Laclare have been quite influential in the solar astrolabe community. It has been claimed that the first visual results that have been obtained at the other astrolabe stations were always different to those obtained simultanously by Laclare at CERGA. Nevertheless, during the course of time there is an evolution of the results that makes them finally converge to the results of CERGA (Chollet 1995; Sinceac et al. 1998). However, such evolution has never been observed in the results of Santiago. On the other hand, according to Chollet \& Sinceac (1999),

“... the apparent solar radius variation is identical for all measures done with astrolabes (except Santiago), and in anticorrelation with the magnetic cycle of the Sun."

We do not share this assertion since as far as we know, before 1999 the only long term data set with results produced simultaneously with those of Santiago was that of CERGA. The other astrolabe stations that could be compared with the results of Santiago did not have a sufficient extension in time to reveal a long term trend in their radius measurements. However, at the present time the results with the CCD astrolabe of the Tubitak National Observatory, Antalya, Turkey, available at the home page of this observatory, for the period 1999.5 to 2001.5 give a radius variation quite consistent with the results of Santiago (see Fig. 4). Concerning the CCD astrolabe of Rio de Janeiro, its results for 1997-2000 are now available at CDS (Jilinski et al. 1999; Puliaev et al. 2000; Penna et al. 2002). It is true that for this period the drift of the radius mesurements of this astrolabe is similar to the results of Laclare. However, it will be shown in this paper that there is a lack of homogeneity in the database from Rio de Janeiro. If a procedure of homogenization is applied, the solar radius observed from Rio de Janeiro shows a variation in time almost identical to the visual results of Santiago.

\section{Solar radius measurements at Rio de Janeiro, 1997-2000}

In a previous paper we discussed the solar radius measurements made at Rio de Janeiro and Santiago between 1997 and 1999 (Noël 2001). However, modifications introduced after our discussion in the results of the CCD astrolabe of Rio de Janeiro (Penna 2002) and the publication of the latest results of this astrolabe (Penna et al. 2002), provide new and quite interesting information for the problem of solar radius variations and give additional evidence that the apparent radius of the Sun varies in phase with solar magnetic activity.

In Fig. 2 we plot the daily mean values of the solar radius obtained with the CCD astrolabe of Rio de Janeiro during the period 1997-2000. These results can be obtained in electronic form at CDS and according to Penna et al. (2002) they

“... supersede all others based on preliminary data from this program."

Therefore, the data of Rio de Janeiro that we shall discuss here are definitive.

The curve in Fig. 2 is a least squares second order fit with a standard deviation of \pm 0 ! 31 . It shows a slowly decreasing trend of the solar radius during 1997-2000. Since the solar maximum of cycle 23 was probably around 2000.5 , the solar radius observed at Rio de Janeiro would have a variation in time of opposing phase with solar activity over these four years. However, the set of points in Fig. 2 give the impression that there are two discontinuities or decreasing jumps in the values of the solar radius around 1998.0 and 1999.0. To verify this impression, we applied least squares linear fits to each set of annual points in Fig. 2. These fits are displayed in Fig. 3A and with its parameters given in Table 1, we obtained the following discontinuities of the radius values:
1998.0
1999.0
2000.0
$-0 . .323 \pm 0 . ' 089$
$+0{ }^{\prime} 063 \pm 0 .{ }^{\prime} 089$.

$-0 . ' 660 \pm 0 . ' 136$

These figures confirm the significant and quite large discontinuities at 1998.0 and 1999.0 in the solar radius observed with the CCD astrolabe of Rio de Janeiro.

It is quite difficult to imagine that these sudden jumps could be due to a systematic effect. We think that it is rather probable that they are due to changes in the instrumental system of 


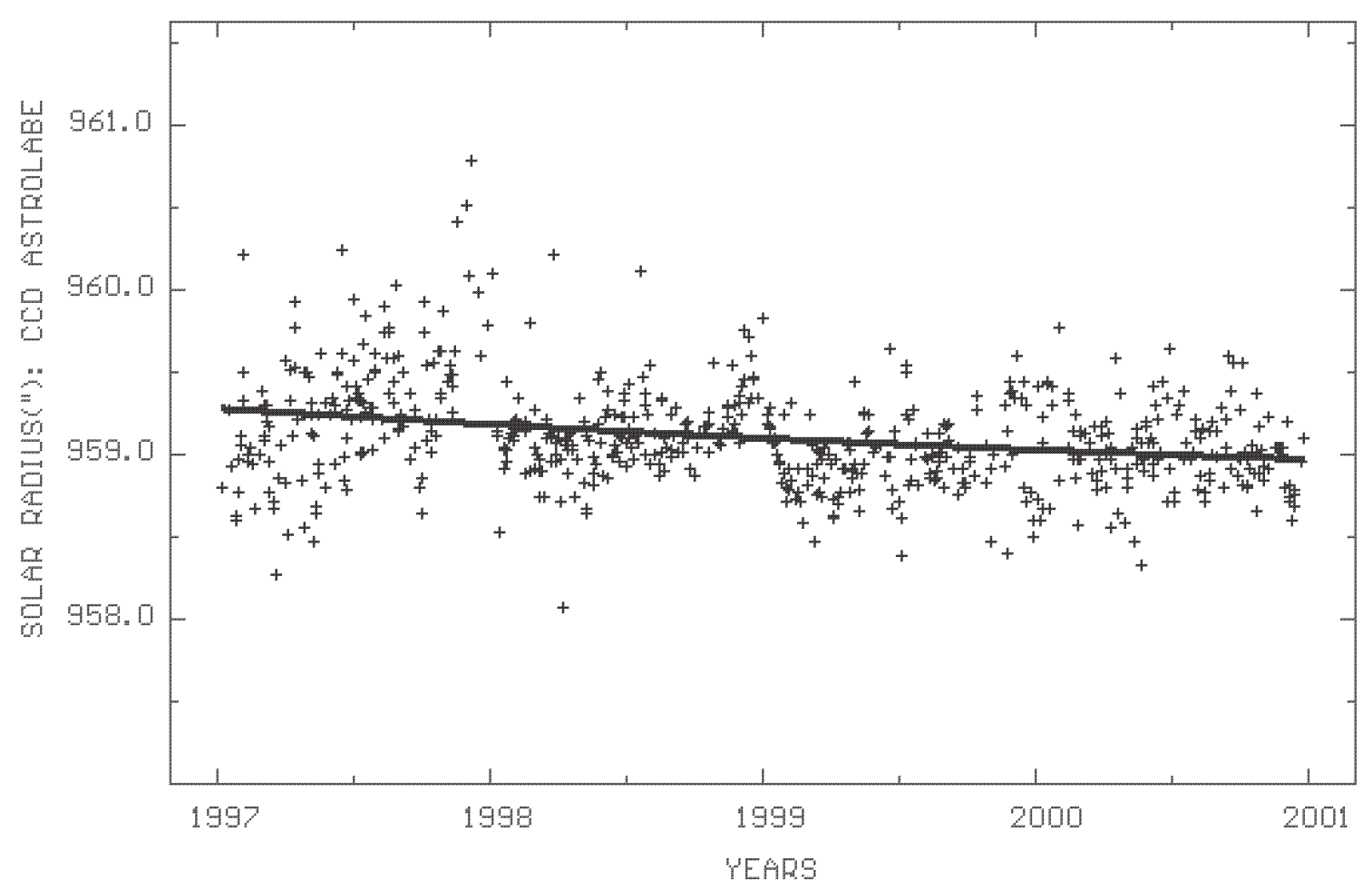

Fig. 2. Daily mean values of solar radius measurements made during 1997-2000 with the CCD astrolabe of Rio de Janeiro. The smoothing curve is a least squares second order fit with a standard deviation of \pm 0 '. 31 . Apparent discontinuities in the radius values can be seen around 1998.0 and 1999.0.

Table 1. Parameters of annual linear fits applied to solar radius measurements with the CCD astrolabe of Rio de Janeiro during 1997-2000. $A$ and $B$ are the coefficients in the equation of the least square fitted lines and $\sigma$ is the standard deviation of the linear fits. $\mathrm{n}$ is the number of daily mean values of the solar radius involved in each fit.

\begin{tabular}{|c|c|c|c|c|c|c|}
\hline Year & \multicolumn{2}{|c|}{$A$} & \multicolumn{2}{|c|}{$B$} & $\bar{\sigma}$ & $\bar{n}$ \\
\hline 1997: & $958 . .920$ & $=0.063$ & 0.746 & 0.114 & \pm 0.358 & 157 \\
\hline 1998 : & 959.005 & 0.039 & 0.260 & 0.071 & 0.251 & 166 \\
\hline 1999 : & 958.943 & 0.037 & 0.071 & 0.069 & 0.245 & 154 \\
\hline 2000 & 959.077 & 0.041 & -0.113 & 0.073 & 0.248 & 152 \\
\hline
\end{tabular}

the CCD astrolabe of Rio de Janeiro. Actually, at least one of these jumps coincides with a modification introduced in this instrument. Indeed, according to Jilinski et al. (1999), on January $1 \mathrm{st}, 1998$, the filter after the image vehicle of the astrolabe of Rio de Janeiro was replaced by one with a narrower bandpass (see also Noël 2001). Since solar radius measurements with CCD cameras are very sensitive to instrumental modifications (Wittmann et al. 2000; Noël 2001), in our opinion it is obvious that the jump in the radius values in 1998.0 and the filter change must be closely related. Concerning the discontinuity around 1999.0, we did not find any information in the publications of Rio de Janeiro about instrumental modification at that epoch. Nevertheless, it being so similar to that of 1998.0 we consider that it was also due to changes in the instrumental system.

Given the large and sudden jumps in the solar radius observed with the astrolabe of Rio de Janeiro associated with at least one modification of its instrumental system, we think that it is not possible to consider these observational results as a homogeneous or internally consistent data set. However, with the computed values of the discontinuities at 1998.0 and 1999.0 given above, it could be possible, at least as a test, to homogenize the data set in order to study the behaviour of the homogeneous values. The results of this test can be seen in Fig. 3B which shows the solar radius observed after removing the jumps of 1998.0 and 1999.0 by applying as corrections the values of both jumps. A least square second order fit now gives a standard deviation of \pm 0.28 , which is an improvement of around $10 \%$ in the internal consistency of the results. On the other hand, the smoothing curve shows its maximum values around year 2000, which is in agreement with a variation in time of the solar radius in phase with solar magnetic activity.

\section{Discusion}

In Fig. 4 we plot monthly mean values of the solar radius given by the astrolabes of Rio de Janeiro, Antalya and Santiago. The monthly means are represented by the center of circles with diameters equal to their mean errors. For Rio de Janeiro they were computed from the homogeneous data set discussed above, and for Antalya from the individual daily measurements that are available at the home page of Tubitak Observatory (Golbasi et al. 2001). On average, each monthly mean is based on 273 and 43 CCD observations for Rio de Janeiro and Antalya respectively, and on 38 visual observations for Santiago. These figures and the dispersion of the circles and the magnitude of its diameters in Fig. 4 do not show higher precision of the CCD 


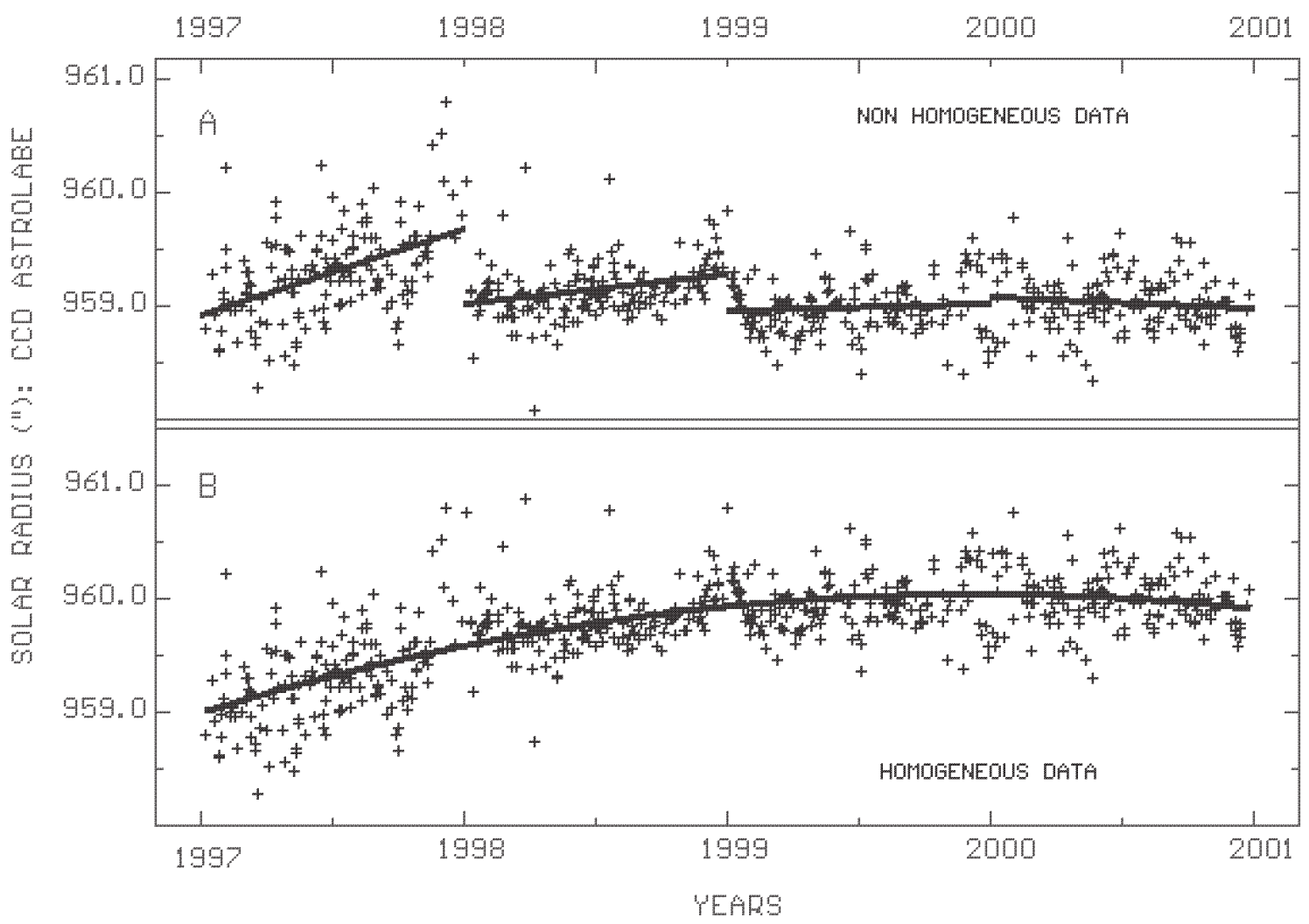

Fig. 3. A). Least squares annual linear fits applied to the solar radius measurements with the CCD astrolabe of Rio de Janeiro. The parameters of these fits are given in Table 1. The origin of the discontinuities around 1998.0 and 1999.0 are discussed in the text. B). The same data displayed in A after a procedure of homogenisation (see text). The smoothing curves is a least squares second order fit with a standard deviation of \pm 0 '. 28 . The maximum values of the curve coincide approximately with the maximum of solar cycle 23 .

astrolabes over the visual one. For further discussions about visual and CCD observations of the solar radius see Wittmann (1997), Wittmann \& Bianda (2000) and Noël (2001) (see also Laclare et al. 1999).

Considering that the observational period at Antalya is rather short in comparisson with those of the other two astrolabes, we have plotted also the curve of a least squares second order fit applied to the set of individual observations at Antalya. This fit, with a standard deviation of \pm 0 '. 36 , gives a similar drift of the solar radius as that shown by the astrolabes of Rio de Janeiro and Santiago.

The curve in Fig. 4 that represents the variation of sunspot number is based on the smoothed monthly means of sunspot number available at the home page of SIDC.

There is a clear agreement between the variations in time of the homogeneous solar radius observed at Rio de Janeiro, the solar radius observed at Santiago and Antalya and the sunspot number variation. From our point of view, it is quite difficult to believe that the improvement of the internal consistency of the results of Rio de Janeiro after our procedure of homogenization, and its agreement with the results of Santiago and Antalya, could be due only to coincidence. Rather, we think that the data displayed in Fig. 3B show the real variation in time of the solar radius observed at Rio de Janeiro.

Figure 4 shows also that there are significant systematic differences between the mean values of the solar diameter obtained by the three astrolabes. The mean values for the periods of observations displayed in Fig. 4 are:

$\begin{array}{ccc}\text { Antalya } & \text { Rio de Janeiro } & \text { Santiago } \\ 958^{\prime \prime} 57 \pm 0.01 & 959.56 \pm 0.01 & 960.56 \pm 0.01 .\end{array}$

Since the astrolabes of Antalya and Rio de Janeiro are equipped with CCD cameras, their results are independent of the observer. Therefore, one should expect a rather low difference in their mean results and not the large difference that they actually give. In our view, this confirms that solar radius measurements with CCD cameras are strongly affected by effects not intrinsic to the Sun (Wittmann 1997, 2000; Noël 2001). The results are very sensitive to instrumental modifications, as shown in Fig. $3 \mathrm{~A}$ by the large jump in solar radius observed with the CCD astrolabe of Rio de Janeiro after a change in its instrumental system.

\section{Conclusions}

The assertion of Chollet and Sinceac (1999) mentioned in Sect. 1, that all solar astrolabes, except Santiago, give identical variations in time of the solar radius and in opposite phase with Sun's magnetic activity, is at issue with what we have shown in this paper. In fact, according to our results, the CCD astrolabes of Rio de Janeiro and Antalya, and the visual astrolabe of Santiago, give almost identical variations in time of the solar radius correlated with the variation of sunspot numbers. 


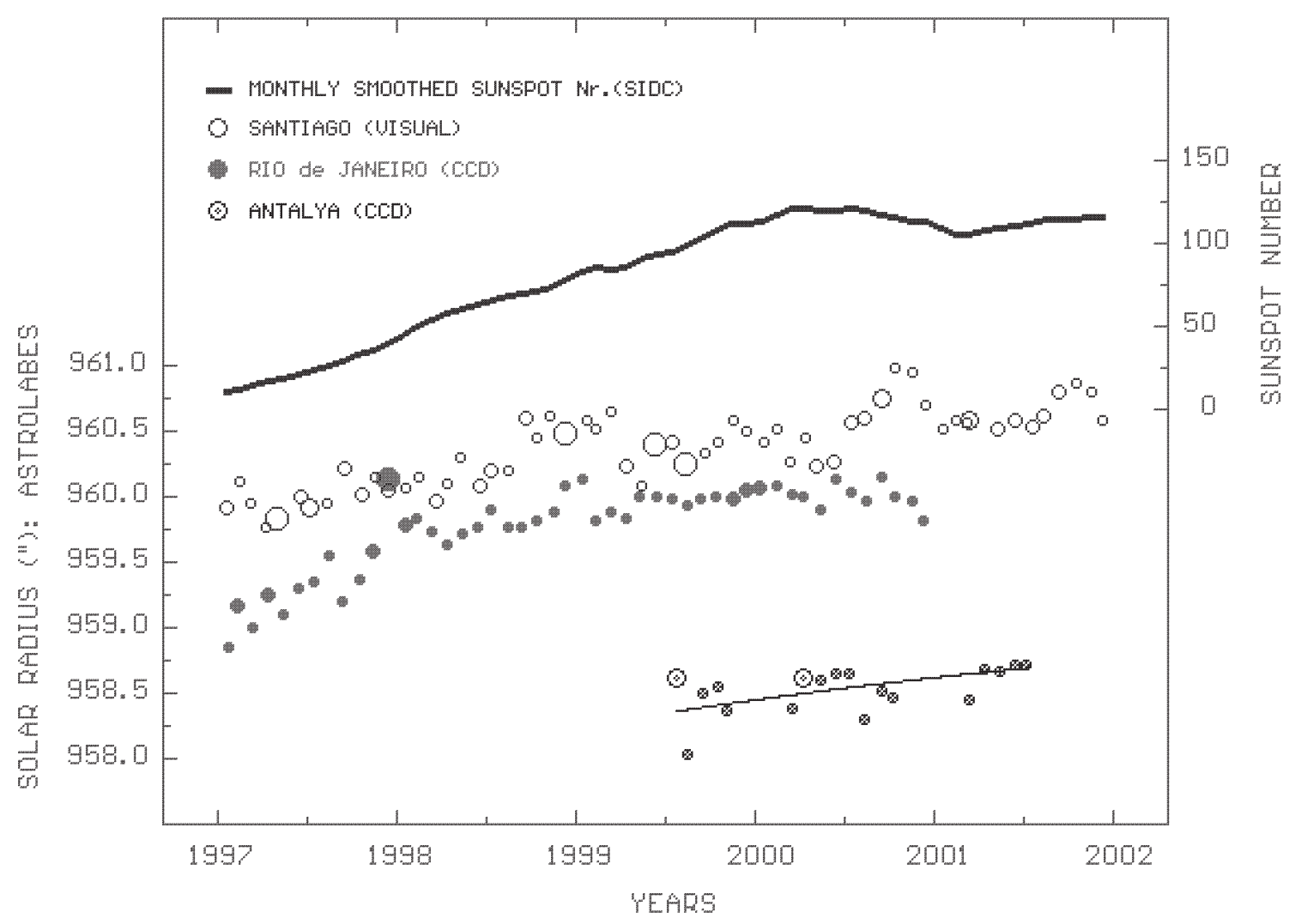

Fig. 4. Monthly means of solar radius obtained with the CCD astrolabes of Antalya and Rio de Janeiro, and with the visual astrolabe of Santiago, and smoothed monthly means of sunspot numbers obtained from SIDC. The monthly values of the solar radius are represented by the center of circles with diameters equal to their mean errors. The smoothing curve for Antalya is a least squares second order fit $(\sigma= \pm 0$ ' 36) applied to individual solar radius measurements made with a CCD astrolabe.

The exception is the astrolabe of CERGA which according to Fig. 1, and following the analysis of Pap et al. (2001), gives a rather marginal solar radius variation in opposite phase with the solar cycle. On the other hand, concerning solar radius measurements made with other instruments, it should be noted that results obtained by Wittmann \& Bianda (2000) between 1990 and 2000 did not show any longterm variation in excess of \pm 0 .' 05 . This was confirmed by the latest results of these authors made until April 26, 2002 (Wittmann 2002).

We did not include in this discussion the CCD astrolabes of San Fernando (Spain) and São Paulo (Brazil), since we do not know long term data of these astrolabes obtained during the observational period of the astrolabe of Santiago.

Finally we would like to emphasize that the agreement of the astrolabes of Antalya, Rio de Janeiro and Santiago, is supported by recent results based on different observational techniques that give also empirical evidence that the apparent solar radius would vary in phase with the cycle of the Sun. For instance:

- From observations made at Mount Wilson Observatory during cycles 21 and 22, following a method described by LaBonte \& Howard (1991), Ulrich \& Bertello (1995) have shown that the apparent radius of the Sun viewed in the spectral line of neutral iron at $525 \mathrm{~nm}$ varies in phase with solar activity.
- Based on radio-observations of the Sun made at $48 \mathrm{GHz}$ during 1991-1993 at Itapetinga Radio Observatory, São Paulo, Brazil, Costa et al. (1999) have detected a decrease of the measured radius as the solar cycle declined.

- In a three year experiment (1996-1998) to detect solar radius fluctuations with the Michelson Doppler Imager (MDI) on board the Solar and Heliospheric Observatory (SOHO), Emilio et al. (2000) found that the solar radius increases approximately in a linear way with the number of sunspots (see also Gough 2001).

- An analysis made by Basu (1998) of homogenised data sets of solar radius measurements made during the last three centuries gives a statistically significant relationship between larger diameters of the Sun and higher levels of solar activity.

Acknowledgements. Our thanks to Dr. Fredrik Rantakyro, Department of Astronomy, Universidad de Chile, for his kind assistance concerning the use of facilities for PostScripts graphics.

\section{References}

Anguita, C., \& Noël, F. 1969, AJ, 74, 954

Basu, D. 1998, Sol. Phys., 183, 291

Chollet, F. 1995, private communication

Chollet, F., \& Noël, F. 1993, A\&A, 276, 655

Chollet, F., \& Sinceac, V. 1999, A\&AS, 139, 219

Costa, J. E. R., Silva, A. V. R., Makhmutov, V. S., et al. 1999, ApJ, 520, L63 
Danjon, A. 1960, in Telescopes, ed. G. P. Kuiper, \& B. M. Middlehurst (The University of Chicago Press), 115

Débarbat, S. 1968, Bull. Astron., 3, 415

Débarbat, S., \& Guinot, B. 1970, La méthode des hauteurs égales en astronomie (Gordon and Breach, Paris)

Emilio, M., Kuhn, J. R., Bush, R. I., \& Scherrer, P. H. 2000, ApJ, 543, 1007

Fricke, W. 1972, ARA\&A, 10, 107

Golbasi, O., Chollet, F., Kilic, H., et al. 2001, A\&A, 368, 1077

Gough, D. 2001, Nature, 410, 313

Jilinski, E. G., Puliaev, S., Penna, J. L., Andrei, A. H., \& Laclare, F. 1999, A\&AS, 135, 227

LaBonte, B. A., \& Howard, R. H. 1981, Science, 214, 907

Laclare, F. 1983, A\&A, 125, 200

Laclare, F., Delmas, C., Coin, J. P., \& Irbah, A. 1996, Sol. Phys., 166, 211

Laclare, F., Delmas, C., Sinseac, V., \& Chollet, F. 1999, C. R. Acad. Sci. Paris, 327, Ser. IIb, 645

Leister, N. V., \& Benevides Soares, P. 1990, C. R. Acad. Sci. Paris, 313, Ser. II, 399

Noël, F. 1987, A\&AS, 68, 219

Noël, F. 2001, A\&A, 374, 697

Noël, F. 2002, in The Shape of the Sun, V Summer School on Solar Astrophysics, Saint Pierre d'Oléron, France, ed. J. P. Rozelot, Lectures Notes in Astrophysics (Springer-Verlag), in press
Pap, J., Rozelot, J. P., Godier, S., \& Varadi, F. 2001, A\&A, 372, 1005 Penna, J. L. 2002, private communication

Penna, J. L., Jilinski, E. G., Andrei, A. H., Puliaev, S. P., \& Reis Neto, E. 2002, A\&A, 384, 650

Puliaev, S., Penna, J. L., Jilinski, E. G., \& Andrei, A. H. 2000, A\&AS, 143,265

Ribes, E., Beardsley, B., Brown, T. M., et al. 1991, in The Sun in Time, ed. C. P. Sonett, M. S. Giampapa, \& M. S. Matthews (The University of Arizona Press), 59

Sánchez, M., Moreno, F., \& Soler, M. 1993, A\&A, 280, 333

Sinceac, V., Chollet, F., Laclare, F., \& Delmas, C. 1998, A\&A, 128, 605

Spruit, H. C. 1994, in The Solar Engine and its Influence on Terrestrial Climate, ed. E. Nesme-Ribes, NATO ASI Ser., V125 (SpringerVerlag, Berlin Heidelberg), 107

Standish, E. M., Débarbat, S., \& Sánchez, M. 1981, A\&A, 102, 371

Ulrich, R. K., \& Bertello, L. 1995, Nature, 377, 214

Vondrak, J. 1969, Bull. Astron. Inst. Czech., 20, 349

Wittmann, A. D. 1997, Sol. Phys., 171, 231

Wittmann, A. D., \& Bianda, M. 2000, in Proc. 1st Solar \& Space Weather Euroconference, The Solar Cycle and Tesrrestrial Climate (ESA SP-463, December 2000), 113

Wittmann, A. D. 2002, private communication 